\title{
Delineamento epidemiológico dos casos notificados de 2008 a 2018 de HIV em pessoas idosas no estado de Pernambuco - Brasil
}

\author{
Epidemiological delineation of reported cases from 2008 to 2018 of HIV in older people in \\ Pernambuco state - Brazil
}

\section{Delineación epidemiológica de casos notificados de 2008 a 2018 de VIH en personas mayores en el estado de Pernambuco - Brasil}

Julianne Damiana da Silva Vicente ${ }^{1 *}$, Camila de Farias Dantas ${ }^{2}$, Liniker Scolfild Rodrigues da Silva ${ }^{1}$, Kelly Regina Machado de Lima², Juliane Raquel Miranda de Santana ${ }^{1}$, Leonia Moreira Trajano $^{1}$, Julianna Carolina da Silva Vicente ${ }^{1}$, Márcia Andrea Oliveira da Cunha ${ }^{1}$.

\section{RESUMO}

Objetivo: Este estudo tem por objetivo delinear o perfil epidemiológico dos casos notificados de HIV em pessoas idosas no período de 2008 a 2018, no estado de Pernambuco, Brasil. Métodos: Trata-se de um estudo epidemiológico descritivo, com abordagem quantitativa, tendo como população e amostra a população idosa do estado de Pernambuco notificada para infecção do vírus HIV entre os anos de 2008 a 2018. Os dados foram coletados através do Sistema de Informação de Agravos de Notificação (Sinan) de Pernambuco, a tabulação dos dados foi realizada por meio do tabulador Tabwin. O estudo foi aprovado por Comitê de Ética em Pesquisa. Resultados: Pode-se observar que o maior percentual de casos de HIV em pessoas idosas no estado de Pernambuco no período de 2008 a 2018, está concentrado na I Região de Saúde, com $69,2 \%$ e no sexo masculino com $63,8 \%$. Cerca de $88 \%$ das pessoas notificadas residem em área urbana. Verificou-se que houve um crescimento significativo dos casos de HIV na pessoa idosa entre os anos estudados. Conclusão: É fundamental que sejam elaboradas novas medidas de prevenção a esta IST, possibilitando ao idoso tornar-se protagonista do autocuidado e incorporando este assunto à rotina dos serviços de saúde.

Palavras-chave: HIV, Idoso, Perfil de saúde.

\section{ABSTRACT}

Objective: This study aims to outline the epidemiological profile of notified cases of HIV in elderly people from 2008 to 2018, in the state of Pernambuco, Brazil. Methods: This is a descriptive epidemiological study, with a quantitative approach, using as population and sample the elderly population of the state of Pernambuco notified for infection of the HIV virus between the years 2008 to 2018. Data were collected through the Information System of Notifiable Diseases (Sinan) of Pernambuco, the tabulation of data was performed using the Tabwin tab. The study was approved by the Research Ethics Committee. Results: It can be observed that the highest percentage of HIV cases in elderly people in the state of Pernambuco in the period from 2008 to 2018, is concentrated in the I Health Region, with $69.2 \%$ and in the male gender, with $63.8 \%$. About $88 \%$ of the notified people live in an urban area. It was found that there was a significant increase in HIV cases in the elderly between the years studied. Conclusion: It is essential that new preventive measures are developed for this STI, enabling the elderly to become the protagonist of self-care and incorporating this issue into the routine of health services.

Keywords: HIV, Elderly, Health profile.

1 Universidade de Pernambuco (UPE), Recife - PE. *E-mail: julianne_vicentte@hotmail.com

2 Instituto Aggeu Magalhães, Fundação Oswaldo Cruz (IAM/FIOCRUZ), Recife - PE. 


\section{RESUMEN}

Objetivo: Este estudio tiene como objetivo delinear el perfil epidemiológico de los casos notificados de VIH en personas mayores de 2008 a 2018, en el estado de Pernambuco, Brasil. Métodos: Se trata de un estudio epidemiológico descriptivo, con enfoque cuantitativo, utilizando como población y muestra a la población anciana del estado de Pernambuco notificada por infección por el virus del VIH entre los años 2008 a 2018. Los datos fueron recolectados a través del Sistema de Información de Enfermedades Notificables (Sinan) de Pernambuco, la tabulación de los datos se realizó utilizando la pestaña Tabwin. El estudio fue aprobado por el Comité de Ética en Investigación. Resultados: Se puede observar que el mayor porcentaje de casos de VIH en ancianos en el estado de Pernambuco en el período de 2008 a 2018, se concentra en la I Región de Salud, con $69,2 \%$ y en el género masculino, con $63,8 \%$. Aproximadamente el $88 \%$ de las personas notificadas viven en una zona urbana. Se encontró que hubo un aumento significativo de casos de VIH en ancianos entre los años estudiados. Conclusión: Es fundamental que se desarrollen nuevas medidas preventivas para esta ITS, que permitan a las personas mayores convertirse en protagonistas del autocuidado e incorporen este tema en la rutina de los servicios de salud.

Palabras clave: $\mathrm{VIH}$, Ancianos, Perfil de salud.

\section{INTRODUÇÃO}

O crescimento da população idosa no Brasil e no mundo é algo relevante nas pesquisas. De acordo com a Organização Mundial da Saúde (OMS), idoso é todo indivíduo com 60 anos ou mais. O Brasil tem mais de 28 milhões de pessoas nessa faixa etária, número que representa $13 \%$ da população do país. E esse percentual tende a dobrar nas próximas décadas, segundo a Projeção da População, divulgada em 2018 pelo IBGE (BRASIL, 2019). Em conformidade com o Art. 1ํ da Lei ํㅜ 10.741, de 1ํ de outubro de 2003, que institui o Estatuto do Idoso, é considerado idoso todo cidadão acima de 60 (sessenta) anos (BRASIL, 2003).

A velhice é, portanto, um processo natural, mas tão plural quanto as diversas culturas humanas existentes e ainda diverso de acordo com a experiência vivida por cada indivíduo. Processo esse que supera em muitas alterações fisiológicas próprias de desgastes do organismo ao longo da vida e da perda de funcionalidades (FERREIRA VHS, et al., 2020).

O processo de envelhecer vem provocando sucessivas mudanças aos setores da economia, política e saúde. De acordo com Veras RP e Oliveira M (2018) um dos maiores feitos da humanidade foi a ampliação do tempo de vida, que se fez acompanhar da melhora substancial dos parâmetros de saúde das populações, ainda que essas conquistas estejam longe de se distribuir de forma equitativa nos diferentes países e contextos socioeconômicos.

Perante a este grande fenômeno, juntamente ao aumento de doenças crônicas, a saúde pública brasileira vem enfrentando diversos desafios. Segundo Viana P, et al. (2017), a faixa etária dos idosos, nos últimos anos, tem constituído foco de preocupação quanto ao aumento da incidência de casos de HIV/Aids. Além disso, estudos focados na investigação do comportamento sexual em indivíduos idosos apontam menor conscientização quanto aos fatores de risco e estratégias de prevenção.

A diminuição da atividade sexual no envelhecimento pode ser interpretada tanto como um fenômeno biológico (próprio do processo de envelhecimento) como uma disfunção sexual, ocasionada pela complexidade de fatores envolvidos nesse período da vida. Desse modo, é preciso trabalhar o fato de que a expressão da sexualidade não termina com o envelhecimento (MACIEL M e LAGANÀ L, 2014).

Ainda que exista no imaginário popular a crença de que as pessoas idosas são menos interessadas por sexo, e por isso não são acometidas pelas Infecções Sexualmente Transmissíveis (ISTs), um estudo de Soares KG e Meneghel SN (2020) mostra que os idosos do sexo masculino enunciam um conceito de sexualidade pautado na satisfação biológica e no ato sexual, enquanto as mulheres valorizam mais a parceria, o afeto e o carinho. Estas doenças estão presentes nesta população, e a epidemia da AIDS ainda se constitui como um importante problema de saúde pública e de enfrentamento ao Sistema Único de Saúde (SUS). 
No estado de Pernambuco, em consonância com o último Boletim Epidemiológico de HIV/Aids do ano de 2018, de julho de 2014 a dezembro de 2017, foram notificados 8.126 casos de HIV. O estado, em 2015, registrou 2.223 casos e uma taxa de detecção (T.D.) de 23,79 por 100mil habitantes (hab.). Em 2017, identificou-se um incremento de $21,32 \%$ dos casos, quando estes chegaram a 2.697 , e de $19,68 \%$ na taxa (28,47/ $100 \mathrm{mil} / \mathrm{hab}$.) (BRASIL, 2018).

$\mathrm{O}$ aumento de casos notificados nas pesquisas vem confirmando o vírus como uma ameaça à saúde pública e a tendência sugere que, em pouco tempo, o número de idosos contaminados pelo HIV será ampliado significativamente, principalmente, devido à vulnerabilidade física e psicológica, pouco acesso a serviços de saúde, além da invisibilidade com que é tratada sua exposição ao risco, seja por via sexual ou uso de drogas ilícitas (CELEDÔNIO LP e ANDRADE LC, 2014).

De acordo com Silva AO, et al. (2017) o crescimento dos casos de HIV entre os idosos, tem sido associado ao envelhecimento da população brasileira, ao aumento da sobrevida das pessoas portadoras do HIV/Aids e ao acesso a medicamentos para distúrbios eréteis, fator que tem prolongado a atividade sexual de idosos, em associação com a desmistificação do sexo nesta idade.

Desde 1986, com a criação do Programa Nacional de DST/AIDS, o Brasil tem desenvolvido estratégias para a prevenção das IST, entretanto, muito pouco se fez em relação à população de idosos. A escassez de estudos epidemiológicos e campanhas de prevenção, somados à ampliação do período sexual ativo, processos fisiológicos do envelhecimento e aspectos comportamentais, têm refletido na incidência de IST e Síndrome da Imunodeficiência Adquirida (AIDS) nos idosos (DORNELAS NETO J, et al., 2015).

É imprescindível que sejam levantadas novas questões sobre o HIV/Aids, trazendo-as para o campo da prática da saúde, como: as abordagens da sexualidade, e dos direitos humanos, o acesso aos serviços e insumos de prevenção, entre tantas outras que, muitas vezes, não são abordadas pelo setor saúde (VALENTE G, et al., 2013).

Diante disso, esta pesquisa tem por objetivo delinear o perfil epidemiológico dos casos notificados de HIV em pessoas idosas, no período de 2008 a 2018, no estado de Pernambuco, Brasil.

\section{MÉTODOS}

Trata-se de um estudo epidemiológico descritivo, longitudinal, do tipo seccional, com abordagem quantitativa. Realizado no estado de Pernambuco, Brasil. Foram utilizadas variáveis relativas à pessoa: Sexo, faixa etária, raça/cor, escolaridade, município de residência, região de saúde, zona (urbana, rural, periurbana), provável modo de transmissão (vertical, sexual, sanguínea) e ano de diagnóstico. Tendo como população e amostra a população idosa do estado de Pernambuco notificada para infecção do vírus HIV entre os anos de 2008 a 2018.

Foram incluídas no estudo pessoas notificadas para infecção do vírus HIV com 60 anos ou mais, no Estado de Pernambuco, do período de 1ำ de janeiro de 2008 a 31 de dezembro de 2018. E, excluídas gestantes, menores de 13 anos e adultos com menos de 60 anos notificados para infecção do vírus HIV, do período de $1^{\circ}$ de janeiro de 2008 a 31 de dezembro de 2018. Bem como, pessoas com mais de 60 anos que não foram notificadas para infecção do vírus HIV no período analisado, no estado de Pernambuco, Brasil.

Os dados foram coletados através do Sistema de Informação de Agravos de Notificação (SINAN) de Pernambuco. A tabulação dos dados foi realizada por meio do tabulador Tabwin e transferida para o programa Microsoft Excel, onde foram distribuídos em planilhas e dispostos em tabelas e gráficos, de acordo com as variáveis selecionadas no estudo e com as 12 Regiões de Saúde do Estado de Pernambuco, para serem analisados.

O projeto de pesquisa foi submetido ao Comitê de Ética e Pesquisa (CEP) da Universidade de Pernambuco (UPE/PROPEGE) para apreciação e parecer, de acordo com a Resolução no 466/12 que aprova as diretrizes e normas regulamentadoras de pesquisas envolvendo seres humanos, e a Resolução no 510/16 que dispõe sobre as normas aplicáveis a pesquisas em Ciências Humanas e Sociais, nas quais também se inserem as pesquisas em saúde. O mesmo foi aprovado com o CAAE: 16691219.2.0000.5207, e no do parecer: 3.509.632. 


\section{RESULTADOS E DISCUSSÃO}

Diante do que foi observado, muitos aspectos precisam ser discutidos. Após a coleta e análise dos dados, pode-se observar que o maior percentual de casos de HIV em pessoas idosas no estado de Pernambuco no período de 2008 a 2018, está concentrado na I Região de Saúde, com 69,2\% (Tabela 1). E isto era algo esperado, uma vez que a mesma concentra os municípios da região metropolitana com o maior número de habitantes.

Por sua vez, o segundo lugar ficou com a IV Região de Saúde (9,57\%), e a X Região apresentou a menor porcentagem, com apenas $0,10 \%$ dos casos. Outras regiões não apresentaram casos notificados ou registraram poucos casos durante os anos analisados, indicando desta forma, possíveis casos de subnotificação.

Tabela 1 - Distribuição dos casos de HIV em pessoas idosas no estado de Pernambuco, de acordo com as Regiões de Saúde, no período de 2008 a 2018.

\begin{tabular}{|c|c|c|c|c|c|c|c|c|c|c|c|c|c|}
\hline \multirow{2}{*}{$\begin{array}{l}\text { Regiões } \\
\text { de Saúde }\end{array}$} & \multicolumn{11}{|c|}{ Ano } & \multirow[t]{2}{*}{ Total } & \multirow[t]{2}{*}{$\%$} \\
\hline & 2008 & 2009 & 2010 & 2011 & 2012 & 2013 & 2014 & 2015 & 2016 & 2017 & 2018 & & \\
\hline I & 18 & 27 & 30 & 42 & 44 & 61 & 61 & 82 & 74 & 98 & 114 & 651 & $69,25 \%$ \\
\hline II & 2 & 2 & 2 & 0 & 0 & 3 & 3 & 4 & 1 & 3 & 6 & 26 & $2,76 \%$ \\
\hline III & 2 & 0 & 1 & 0 & 5 & 5 & 6 & 10 & 7 & 10 & 10 & 56 & $5,95 \%$ \\
\hline IV & 2 & 2 & 7 & 4 & 5 & 5 & 13 & 14 & 10 & 10 & 18 & 90 & $9,57 \%$ \\
\hline V & 1 & 1 & 4 & 3 & 3 & 4 & 1 & 6 & 5 & 1 & 5 & 34 & $3,61 \%$ \\
\hline VI & 0 & 0 & 1 & 0 & 0 & 2 & 0 & 0 & 4 & 2 & 1 & 10 & $1,06 \%$ \\
\hline VII & 0 & 0 & 1 & 0 & 0 & 2 & 0 & 0 & 1 & 3 & 2 & 9 & $0,95 \%$ \\
\hline VIII & 0 & 1 & 2 & 5 & 1 & 2 & 2 & 0 & 3 & 2 & 4 & 22 & $2,34 \%$ \\
\hline IX & 0 & 0 & 1 & 0 & 0 & 1 & 1 & 1 & 0 & 0 & 3 & 7 & $0,74 \%$ \\
\hline$x$ & 0 & 0 & 0 & 0 & 0 & 0 & 0 & 1 & 0 & 0 & 0 & 1 & $0,10 \%$ \\
\hline XI & 0 & 1 & 0 & 0 & 0 & 1 & 0 & 0 & 4 & 1 & 9 & 16 & $1,70 \%$ \\
\hline XII & 0 & 2 & 0 & 3 & 0 & 3 & 1 & 1 & 4 & 3 & 1 & 18 & $1,91 \%$ \\
\hline TOTAL & $\begin{array}{c}25 \\
2,65 \%\end{array}$ & $\begin{array}{c}36 \\
3,82 \%\end{array}$ & $\begin{array}{c}49 \\
5,21 \%\end{array}$ & $\begin{array}{c}57 \\
6,06 \%\end{array}$ & $\begin{array}{c}58 \\
6,17 \%\end{array}$ & $\begin{array}{c}89 \\
9,46 \%\end{array}$ & $\begin{array}{c}88 \\
9,36 \%\end{array}$ & $\begin{array}{c}119 \\
12,65 \%\end{array}$ & $\begin{array}{c}113 \\
12,02 \%\end{array}$ & $\begin{array}{c}133 \\
14,14 \%\end{array}$ & $\begin{array}{c}173 \\
18,40 \%\end{array}$ & $\begin{array}{c}940 \\
100 \%\end{array}$ & $\begin{array}{l}100 \% \\
100 \%\end{array}$ \\
\hline
\end{tabular}

Fonte: Vicente JDS, et al., 2021; dados extraídos do Sinan, 2019.

O pensamento estereotipado pode estar relacionado ao alto índice de casos ignorados, em branco e possíveis casos de subnotificações durante os anos estudados, pois muitos profissionais de saúde ainda tratam o sexo e a sexualidade como um tabu, e esta ideia pesa ainda mais quando é referida a um público idoso. Pois, vale registrar ainda, que a realidade de ser idoso e viver com o HIV/Aids se coloca como uma realidade muitas vezes surpreendente, impensada e de difícil aceitação, uma vez que contraria os estereótipos especificamente vinculados aos idosos, principalmente relacionados às concepções de assexualidade nesse momento da vida (CASSETÉ J, et al., 2016).

Corroborando as informações feitas por Casseté J, et al. (2016), um estudo de Aguiar RB, et al. (2020) constatou que existe uma correlação positiva entre os níveis de conhecimento e atitudes em relação à sexualidade na terceira idade. Significa que os indivíduos que possuem mais tempo de diagnóstico tendem a ter mais domínio sobre o assunto e, consequentemente, apresentam atitudes menos conservadoras. Vale ressaltar que um maior conhecimento e atitudes mais permissivas sobre sexualidade do idoso não indica, necessariamente, melhor entendimento sobre a transmissibilidade.

A Tabela 2 consiste na distribuição dos casos de HIV em pessoas idosas no Estado de acordo com a zona de residência. No qual, $88 \%$ destas pessoas notificadas residem em área urbana, todavia, $4,78 \%$ delas moram em zona rural e $0,31 \%$ em zona periurbana. Sendo assim, há a necessidade de garantir os princípios de descentralização e equidade do SUS, para que todos os usuários, especialmente os que apresentam dificuldade de acesso nos municípios de pequeno porte, obtenham informação dos programas de prevenção e atendimento relacionado ao HIV/Aids.

Uma vez que, de acordo com Pereira B, et al. (2014), a resposta social direcionada à prevenção ao HIV/AIDS depende diretamente do acesso dos indivíduos à informação, assim como aos recursos disponíveis para sua proteção, promovidos pelos setores, representados pelas Instituições de referência, face ao controle dos importantes problemas de saúde, na busca da redução das desigualdades e distribuição equitativa do poder, nos diferentes contextos sociais. 
Tabela 2 - Distribuição dos casos de HIV em pessoas idosas no estado de Pernambuco, de acordo com a Zona de Residência, no período de 2008 a 2018.

\begin{tabular}{|c|c|c|c|c|c|c|c|c|}
\hline \multirow{2}{*}{ ZONA } & \multicolumn{2}{|c|}{60 a 69 anos } & \multicolumn{2}{|c|}{70 a 79 anos } & \multicolumn{2}{|c|}{80 anos e mais } & \multicolumn{2}{|c|}{ TOTAL } \\
\hline & № & $\%$ & № & $\%$ & $\mathrm{~N}^{0}$ & $\%$ & № & $\%$ \\
\hline Urbana & 688 & $73,19 \%$ & 111 & $11,80 \%$ & 29 & $3,0 \%$ & 828 & $88,0 \%$ \\
\hline Rural & 40 & $4,25 \%$ & 03 & $0,31 \%$ & 02 & $0,21 \%$ & 45 & $4,78 \%$ \\
\hline Periurbana & 02 & $0,21 \%$ & 01 & $0,10 \%$ & 00 & 00 & 03 & $0,31 \%$ \\
\hline Ignorado & 05 & $0,53 \%$ & 00 & 00 & 00 & 00 & 05 & $0,53 \%$ \\
\hline Em branco & 50 & $5,31 \%$ & 08 & $0,85 \%$ & 01 & $0,10 \%$ & 59 & $6,27 \%$ \\
\hline
\end{tabular}

Fonte: Vicente JDS, et al., 2021; dados extraídos do Sinan, 2019.

Com relação a incidência dos casos (Gráfico 1), houve um crescimento significativo dos casos de HIV na pessoa idosa entre os anos estudados. Em 2008 o coeficiente foi de 3,0 casos/100 mil habitantes, comparado a 2018 com 15,65. Verificou-se, desta forma, um aumento bastante considerável. Pode-se observar, ainda, que a incidência continuou estável ou diminuiu nos anos de 2012, 2014 e 2016 , enfatizando possíveis casos de subnotificação nestes períodos.

A ampliação do número de casos de infecção pelo HIV na população idosa se relaciona a alguns fatores: prolongamento da sobrevida, devido à terapia antirretroviral; escassez de campanhas direcionadas à prática sexual segura entre idosos, como a utilização de preservativos; além da existência de tabu sobre a sexualidade na velhice. Ademais, destaca-se a carência de conhecimento nesse segmento a respeito da patologia pelo próprio idoso (ALENCAR RA, et al., 2018; MAIA DC, et al., 2018).

Gráfico 1 - Coeficiente de Incidência dos casos de HIV em pessoas idosas por ano de notificação no estado de Pernambuco, no período de 2008 a 2018.

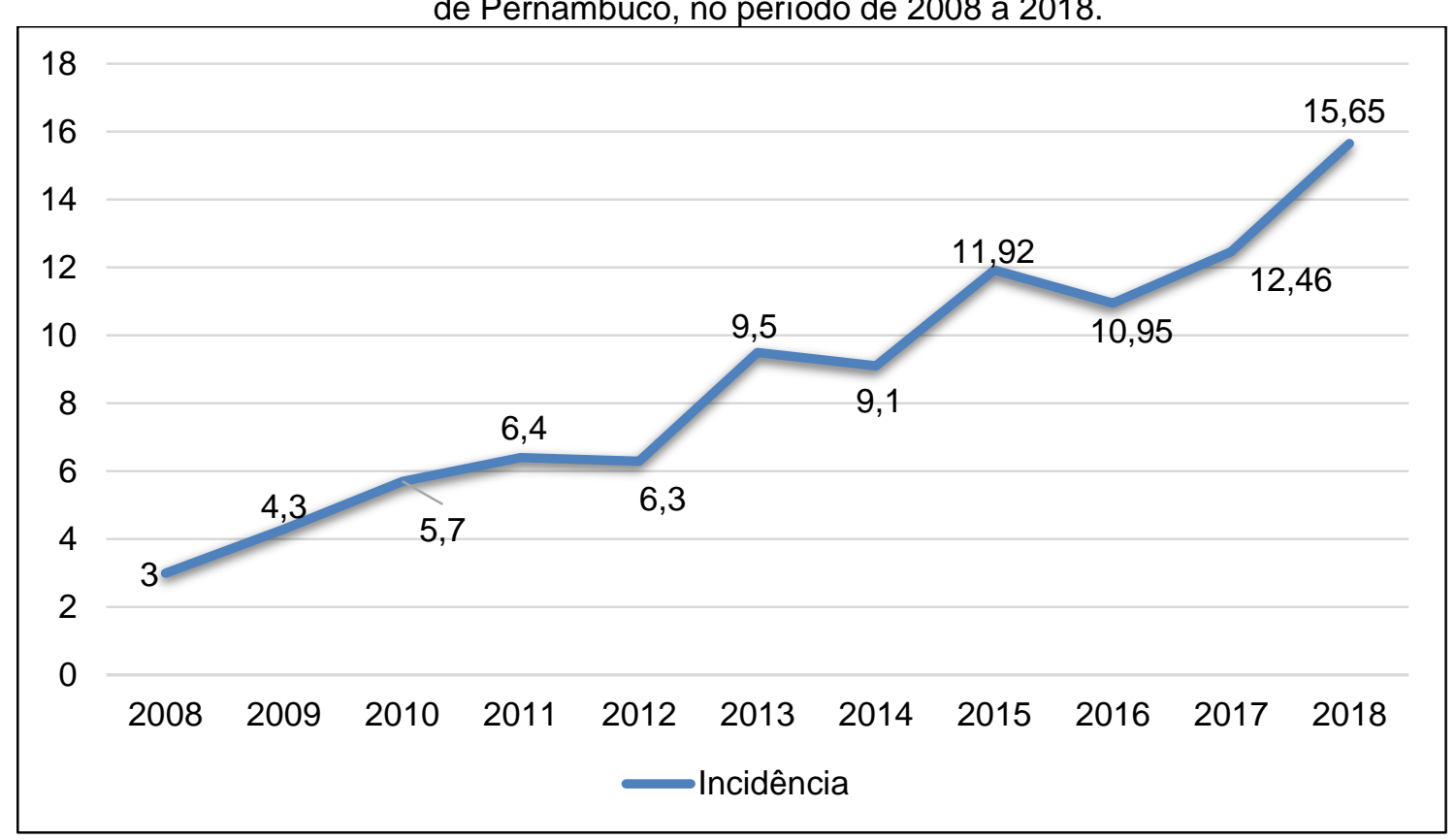

Fonte: Vicente JDS, et al., 2021; dados extraídos do Sinan, 2019.

De acordo com a Tabela 3, o maior número de casos de HIV na terceira idade em Pernambuco, se concentrou no sexo masculino com $63,8 \%$ dos casos, que também coincide com o estudo de Vieira CPB, et al. (2021) e Sacco RCCS, et al. (2020). Por sua vez, o feminino apresentou 36,1\%. Sendo assim, pode-se observar a diferença de percentual entre o número de casos de homens idosos com HIV comparado ao de mulheres idosas infectadas pelo vírus.

E este fator pode ser explicado por diversos aspectos, desde o fato de a mulher ter um maior cuidado com a sua saúde, em que a partir de muito cedo foi incentivada a se cuidar. Bem como, ao desconhecimento dos métodos de prevenção por parte dos homens, e à resistência ao uso do preservativo masculino e sua negociação com as parcerias sexuais, mesmo entre casais sorodiscordantes (BARBOZA $R, 2012$ ). 
Como também, o preconceito e o estigma que se apresenta em potencial, com efeitos sobre a identidade dos indivíduos, dos grupos e das relações sociais, além do reflexo nos processos de saúde e adoecimento (CASSETÉ J, et al., 2016). São condicionantes que enfatizam estes preceitos ainda tão evidentes na sociedade.

Em um estudo realizado por MACHADO YY, et al. (2020) foi apontado divergências nas representações do HIV entre os sexos; maior positividade geral, com elementos do universo reificado e significados associados ao enfrentamento da doença no sexo masculino; e uma tendência mais negativa e acompanhada de elementos de permanência das representações originais do HIV/Aids, com elementos afetivos na representação do grupo do sexo feminino. Isto demonstra o quanto a variável gênero interfere em diversas instâncias do cotidiano em sociedade e, por este motivo, necessita ser bem discutida.

Tabela 3 - Distribuição dos casos de HIV em pessoas idosas no estado de Pernambuco, de acordo com o sexo, escolaridade e raça/cor, no período de 2008 a 2018.

\begin{tabular}{|c|c|c|c|c|c|c|c|c|}
\hline \multirow{2}{*}{$\begin{array}{c}\text { VARIAVÉIS / Pessoas com } 60 \text { anos } \\
\text { e mais }\end{array}$} & \multicolumn{2}{|c|}{60 a 69 anos } & \multicolumn{2}{|c|}{70 a 79 anos } & \multicolumn{2}{|c|}{80 anos e mais } & \multicolumn{2}{|c|}{ TOTAL } \\
\hline & $\mathrm{N}$ & $\%$ & $\mathrm{~N}$ & $\%$ & $\mathrm{~N}$ & $\%$ & $\mathrm{~N}$ & $\%$ \\
\hline \multicolumn{9}{|l|}{ SEXO } \\
\hline Masculino & 501 & $53,29 \%$ & 77 & $8,1 \%$ & 22 & $2,34 \%$ & 600 & $63,82 \%$ \\
\hline Feminino & 284 & $30,21 \%$ & 46 & $4,89 \%$ & 10 & $1,06 \%$ & 340 & $36,17 \%$ \\
\hline \multicolumn{9}{|l|}{ ESCOLARIDADE } \\
\hline Analfabeto & 86 & $9,14 \%$ & 24 & $2,55 \%$ & 01 & $0,10 \%$ & 111 & $11,80 \%$ \\
\hline $1^{\circ}$ a $4^{\circ}$ série incompleta do EF & 107 & $11,38 \%$ & 19 & $2,02 \%$ & 06 & $0,63 \%$ & 132 & $14,04 \%$ \\
\hline $4^{\circ}$ série completa do EF & 61 & $6,48 \%$ & 07 & $0,74 \%$ & 01 & $0,10 \%$ & 69 & $7,34 \%$ \\
\hline $5^{\circ}$ a $8^{\circ}$ série incompleta do EF & 88 & $9,36 \%$ & 10 & $1,06 \%$ & 04 & $0,42 \%$ & 102 & $10,85 \%$ \\
\hline Ensino Fundamental Completo & 49 & $5,21 \%$ & 07 & $0,74 \%$ & 00 & 00 & 56 & $5,95 \%$ \\
\hline Ensino Médio Incompleto & 25 & $2,65 \%$ & 02 & $0,21 \%$ & 02 & $0,21 \%$ & 29 & $3,08 \%$ \\
\hline Ensino médio completo & 71 & $7,55 \%$ & 10 & $1,06 \%$ & 00 & 00 & 81 & $8,61 \%$ \\
\hline Educação Superior Incompleta & 04 & $0,42 \%$ & 00 & 00 & 00 & 00 & 04 & $0,42 \%$ \\
\hline Educação Superior Completa & 46 & $4,89 \%$ & 04 & $0,42 \%$ & 00 & 00 & 50 & $5,31 \%$ \\
\hline Ignorado & 224 & $23,82 \%$ & 34 & $3,61 \%$ & 16 & $1,70 \%$ & 274 & $29,14 \%$ \\
\hline Não se aplica & 00 & 00 & 00 & 00 & 00 & 00 & 00 & 00 \\
\hline Em branco & 24 & $2,55 \%$ & 06 & $0,63 \%$ & 02 & $0,21 \%$ & 32 & $3,40 \%$ \\
\hline \multicolumn{9}{|l|}{ RAÇA/COR } \\
\hline Branca & 147 & $15,63 \%$ & 32 & $3,40 \%$ & 06 & $0,63 \%$ & 185 & $19,66 \%$ \\
\hline Preta & 81 & $8,61 \%$ & 09 & $0,95 \%$ & 01 & $0,10 \%$ & 91 & $9,66 \%$ \\
\hline Amarela & 01 & $0,10 \%$ & 00 & 00 & 01 & $0,10 \%$ & 02 & $0,20 \%$ \\
\hline Parda & 456 & $48,51 \%$ & 69 & $7,34 \%$ & 17 & $1,80 \%$ & 542 & $57,65 \%$ \\
\hline Indígena & 05 & $0,53 \%$ & 00 & 00 & 00 & 00 & 05 & $0,53 \%$ \\
\hline Ignorado & 87 & $9,25 \%$ & 13 & $1,38 \%$ & 07 & $0,74 \%$ & 107 & $11,37 \%$ \\
\hline Em branco & 08 & $0,85 \%$ & 00 & 00 & 00 & 00 & 08 & $0,85 \%$ \\
\hline
\end{tabular}

Fonte: Vicente JDS, et al., 2021; dados extraídos do Sinan, 2019.

No que diz respeito à variável escolaridade, $14,0 \%$ destes idosos estudaram até a 1 ㅇa a $4^{\circ}$ série do ensino fundamental e $11,8 \%$ são analfabetos. Em $29,1 \%$ dos casos este quesito foi ignorado, sendo um aspecto preocupante, uma vez que esta variável corrobora para o entendimento do contexto social que estes indivíduos cresceram e se desenvolveram (Tabela 3).

Deve-se destacar que indivíduos com muitos anos de estudo têm maior acesso aos serviços básicos de saúde e, consequentemente, maior contato com informações relacionadas à sexualidade, além de assimilar melhor as orientações passadas pelos profissionais. Mas, esse fato não exclui a necessidade de fomentar junto aos idosos, principalmente aos que estão em situação de maior vulnerabilidade, ações preventivas e de educação em saúde que favoreçam a reflexão, a mudança de comportamento, a redução da vulnerabilidade e de estigmas frente ao HIV/AIDS e outras IST (AGUIAR RB, et al., 2020).

Em relação à raça/cor, o maior número se concentrou em pessoas idosas de cor parda $(57,65 \%)$, seguidos de cor branca com $19,6 \%$ e preta $9,66 \%$. A indígena apresentou uma porcentagem de apenas $0,53 \%$, e a amarela a menor com $0,20 \%$. Vale salientar que, assim como nas outras, esta variável também apresentou um alto número de ignorado, com 11,3\% dos casos, como demonstrado na Tabela 3. 
É importante destacar o crescimento da população de cor branca acometida pelo HIV. Todavia, Taquette SR e Meirelles ZV (2013) mostram que há argumentos que evidenciam uma maior exposição das mulheres negras a fatores que podem potencializar a susceptibilidade à infecção pelo HIV, entre eles: os individuais (baixa autoestima, atividade sexual precoce, não adoção de práticas sexuais seguras), os sociais (discriminação racial, menor acesso à renda/ emprego/educação/ saúde, violência sexual/gênero) e os coletivos/programáticos (racismo institucional, ausência de políticas públicas direcionadas a este público, etc.).

Oliveira BLCA, et al. (2014) enfatizam a desigual exposição a fatores de risco entre os grupos raciais ao longo dos ciclos de vida, no qual as características individuais e contextuais influenciam a adoção de comportamentos saudáveis ou prejudiciais à saúde. Esses fatores variam entre os grupos raciais e favorecem a ocorrência de diferenças raciais nas estimativas de doenças crônicas, como o é o caso do HIV/Aids.

A Tabela 4 está relacionada ao Provável Modo de Transmissão do HIV, no qual foi dividida em 06 eixos de acordo com a ficha de notificação compulsória de Aids acima de 13 anos. São eles: transmissão vertical, transmissão sexual, uso de drogas injetáveis, tratamento para hemofilia/transfusão, transfusão sanguínea e acidente com material biológico. Em todos os eixos ocorreram um alto número de ignorado, todavia, podese observar que a maioria dos casos foi transmitido por via sexual, resultado também obtido por Viera CPB, et al. (2021), através de relações sexuais com homens $(37,9 \%)$, seguido de relações sexuais com mulheres $(28,7 \%)$.

Tabela 4 - Distribuição dos casos de HIV em pessoas idosas no estado de Pernambuco, de acordo com o provável modo de transmissão do vírus HIV, no período de 2008 a 2018.

\begin{tabular}{|c|c|c|c|c|c|c|c|c|}
\hline \multirow{2}{*}{$\begin{array}{c}\text { PROVÁVEL MODO DE } \\
\text { TRANSMISSÃO / Pessoas com } 60 \\
\text { anos e mais }\end{array}$} & \multicolumn{2}{|c|}{60 a 69 anos } & \multicolumn{2}{|c|}{70 a 79 anos } & \multicolumn{2}{|c|}{80 anos e mais } & \multicolumn{2}{|c|}{ TOTAL } \\
\hline & $\mathrm{N}$ & $\%$ & $\mathrm{~N}$ & $\%$ & $\mathrm{~N}$ & $\%$ & $\mathrm{~N}$ & $\%$ \\
\hline \multicolumn{9}{|l|}{ Transmissão Vertical } \\
\hline SIM & 01 & $0,10 \%$ & 01 & $0,10 \%$ & 01 & $0,10 \%$ & 03 & $0,30 \%$ \\
\hline NÃO & 722 & $76,80 \%$ & 110 & $11,70 \%$ & 28 & $2,97 \%$ & 860 & $91,47 \%$ \\
\hline Ignorado & 62 & $6,59 \%$ & 12 & $1,27 \%$ & 03 & $0,31 \%$ & 77 & $8,17 \%$ \\
\hline \multicolumn{9}{|l|}{ Transmissão Sexual } \\
\hline Relações sexuais com homens & 304 & $32,34 \%$ & 42 & $4,46 \%$ & 11 & $1,17 \%$ & 357 & $37,97 \%$ \\
\hline Relações sexuais com mulheres & 218 & $23,19 \%$ & 43 & $4,57 \%$ & 09 & $0,95 \%$ & 270 & $28,71 \%$ \\
\hline $\begin{array}{l}\text { Relações sexuais com homens e } \\
\text { mulheres }\end{array}$ & 32 & $3,40 \%$ & 02 & $0,21 \%$ & 00 & 00 & 34 & $3,61 \%$ \\
\hline Ignorado & 231 & $24,57 \%$ & 36 & $3,82 \%$ & 12 & $1,27 \%$ & 279 & $29,66 \%$ \\
\hline \multicolumn{9}{|l|}{ Uso de drogas injetáveis } \\
\hline SIM & 02 & $0,21 \%$ & 00 & 00 & 01 & $0,10 \%$ & 03 & $0,31 \%$ \\
\hline NÃO & 476 & $50,63 \%$ & 73 & $7,76 \%$ & 15 & $1,59 \%$ & 564 & $59,98 \%$ \\
\hline Ignorado & 307 & $32,65 \%$ & 50 & $5,31 \%$ & 16 & $1,70 \%$ & 373 & $39,66 \%$ \\
\hline \multicolumn{9}{|c|}{ Tratamento para Hemofilia/Hemotransfusão } \\
\hline SIM & 01 & $0,10 \%$ & 00 & 00 & 00 & 00 & 01 & $0,10 \%$ \\
\hline NÃO & 570 & $60,63 \%$ & 93 & $9,89 \%$ & 19 & $2,02 \%$ & 682 & $72,54 \%$ \\
\hline Ignorado & 214 & $22,76 \%$ & 30 & $3,19 \%$ & 13 & $1,38 \%$ & 257 & $27,33 \%$ \\
\hline \multicolumn{9}{|l|}{ Transfusão sanguínea } \\
\hline SIM & 04 & $0,42 \%$ & 00 & 00 & 01 & $0,10 \%$ & 05 & $0,52 \%$ \\
\hline NÃO & 463 & $49,25 \%$ & 72 & $7,65 \%$ & 13 & $1,38 \%$ & 548 & $58,28 \%$ \\
\hline Ignorado & 318 & $33,82 \%$ & 51 & $5,42 \%$ & 18 & $1,91 \%$ & 387 & $41,15 \%$ \\
\hline \multicolumn{9}{|l|}{ Acidente com material biológico } \\
\hline SIM & 00 & 00 & 00 & 00 & 00 & 00 & 00 & 00 \\
\hline NÃO & 535 & $56,91 \%$ & 75 & $7,97 \%$ & 20 & $2,12 \%$ & 630 & $67 \%$ \\
\hline Ignorado & 250 & $26,59 \%$ & 48 & $5,10 \%$ & 12 & $1,27 \%$ & 310 & $33 \%$ \\
\hline
\end{tabular}

Fonte: Vicente JDS, et al., 2021; dados extraídos do Sinan, 2019.

Um fato inusitado ocorrido durante o estudo, foi a descoberta de 3 (três) registros de casos de transmissão vertical $(0,30 \%)$, indicando desta forma, uma provável falha no preenchimento destas fichas. Havendo assim, a necessidade de uma investigação por se tratar um público idoso (Tabela 4). 
Pois, é improvável idosos acima de 60 anos terem adquirido HIV através de transmissão vertical, visto que o vírus da imunodeficiência adquirida surgiu no Brasil na década de 1980, sendo disseminando desde 1981, e considerado um dos maiores problemas de saúde pública no Brasil e no mundo (SILVA R, et al., 2013).

Ainda na variável "Provável modo de transmissão", ocorreu um baixo número de casos de transmissão do HIV por uso de drogas injetáveis $(0,31 \%)$, tratamento para Hemofilia/Hemotransfusão $(0,10 \%)$, transfusão sanguínea (0,52\%), e acidente com material biológico ( $0 \%$ ) (Tabela 4). Embora estas variáveis tenham apresentado uma menor porcentagem, vale salientar a importância de estes casos serem investigados pela vigilância epidemiológica municipal, e serem acompanhados pelo Estado.

Lazzarotto AR, et al. (2008) relata que o principal fator de risco para DST em idosos é a prática sexual insegura. Com o aumento da idade, existe uma tendência em diminuir o uso de preservativos nas relações sexuais. Dentre os fatores que contribuem para a baixa adesão do uso do preservativo nesta população estão: menor preocupação com concepção (SZIRONY TA, 1999; PEATE I, 2007); dificuldades com o manuseio do preservativo e piora no desempenho sexual (LAROQUE MF et al., 2011). Somam-se ainda, as crescentes exposições a situações de risco, relacionadas ao aumento de divórcio, viuvez, procura de parceiros sexuais na internet e aumento do turismo sexual (MINICHIELLO V, et al., 2012).

Além de se pensar no modo de transmissão, os olhares também devem ser direcionados ao diagnóstico de HIV. Pois, a literatura demonstra que tal aspecto ocorre tardiamente. E, quando se trata dos idosos, o diagnóstico não precoce pode trazer perigos, já que isto permite que o sistema imune se torne cada vez mais comprometido, resultando em aumento de doenças oportunistas e rápida progressão para a AIDS (DORNELAS NETO J, et al., 2015).

E, quanto ao tratamento, Dornelas Neto J, et al. (2015) conseguiu concluir - através de outros pesquisadores - que os efeitos colaterais e as toxicidades resultantes da terapia antirretroviral podem ser esperados com mais frequência em pacientes com mais de 50 anos. Os efeitos mais comuns observados são: desordens no metabolismo de lipídios e glicose, hipertensão, com consequente predisposição a doenças cardiovasculares e cerebrovasculares, além de osteopenia, osteoporose, perda muscular, esteatose hepática, pancreatite, neuropatia periférica e hiperlactatemia, e vários outros distúrbios.

Por fim, a Tabela 5, apresenta a distribuição dos casos de HIV no público idoso de acordo com as Categorias de Exposição Hierarquizadas mais expressivas nas fichas analisadas. O público heterossexual apresentou uma porcentagem de $58,61 \%$, seguido do homossexual com $7,22 \%$ e bissexual com $3,40 \%$. Todavia, mais uma vez, vale destacar o alto número de ignorado, com $29,67 \%$.

Tabela 5 - Distribuição dos casos de HIV em pessoas idosas do sexo feminino e masculino, de acordo com a categoria de exposição, no período de 2008 a 2018 no estado de Pernambuco.

\begin{tabular}{ccccccc}
\hline Categoria de Exposição & Masculino & $\%$ & Feminino & $\%$ & Total & $\%$ \\
\hline Homossexual & 62 & $6,59 \%$ & 6 & $\mathbf{0 , 6 3 \%}$ & $\mathbf{6 8}$ & $\mathbf{7 , 2 2} \%$ \\
Homo/Hemofílico & 1 & $0,10 \%$ & 0 & $\mathbf{0}$ & $\mathbf{1}$ & $\mathbf{0 , 1 0 \%}$ \\
Bissexual & 32 & $3,40 \%$ & 0 & $\mathbf{0}$ & $\mathbf{3 2}$ & $\mathbf{3 , 4 0 \%}$ \\
Bi/Drogas & 1 & $0,10 \%$ & 0 & $\mathbf{0}$ & $\mathbf{1}$ & $\mathbf{0 , 1 0 \%}$ \\
Heterossexual & 261 & $27,76 \%$ & 290 & $\mathbf{3 0 , 8 5 \%}$ & $\mathbf{5 5 1}$ & $\mathbf{5 8 , 6 1 \%}$ \\
Hetero/Droga & 0 & 0 & 2 & $\mathbf{0 , 2 1 \%}$ & $\mathbf{2}$ & $\mathbf{0 , 2 1 \%}$ \\
Perinatal & 3 & $0,31 \%$ & 1 & $\mathbf{0 , 1 0} \%$ & $\mathbf{4}$ & $\mathbf{0 , 4 1 \%}$ \\
Ignorado & 240 & $25,53 \%$ & 39 & $\mathbf{4 , 1 4 \%}$ & $\mathbf{2 7 9}$ & $\mathbf{2 9 , 6 7 \%}$ \\
\hline
\end{tabular}

Fonte: Vicente JDS, et al., 2021; dados extraídos do Sinan, 2019.

Vale salientar ainda que foram notificados casos de transmissão do HIV por uso de drogas, na categoria $\mathrm{Bi} /$ drogas $(0,1 \%)$ e Hetero/drogas $(0,2 \%)$. Em suma, o aumento da população idosa e as demandas relacionadas a persistência de doenças transmissíveis como o HIV/Aids através de uma transição epidemiológica prolongada, representam desafios importantes para o Sistema de Saúde Brasileiro.

Este estudo foi realizado através de dados secundários, o que implica em algumas limitações, devido ao grande número de variáveis ignoradas e em branco, bem como possíveis erros de digitação, 
comprometendo assim a confiabilidade dos dados. Vale destacar que, os dados relacionados ao ano de 2018 estão sujeitos à alteração, podendo sofrer um aumento, uma vez que foram coletados no final do ano de 2019, e neste período o banco de dados do Estado não estava fechado totalmente.

Igualmente, outras pesquisas trazem sobre o problema da subnotificação. Affeldt $A B$, et al. (2015), afirmam que, tamanha subnotificação dos casos compromete a melhor avaliação do perfil epidemiológico desses pacientes, prejudicando o planejamento de medidas de prevenção primária voltadas às faixas etárias e grupos mais expostos à infecção.

A partir desta pesquisa pode-se entender a questão do HIV em pessoas idosas em diversos contextos, configurados enquanto fenômeno social de extensa dimensão, moldados por princípios éticos, morais e religiosos, que impactam de forma significativa nas ações e políticas no âmbito da saúde pública.

\section{CONCLUSÃO}

Com o crescimento da população idosa, urge a adoção de políticas de saúde específicas para este grupo, dentre as quais se incluem ações eficazes na prevenção aos casos de HIV. O desafio que se impõe, no âmbito da saúde pública, é para que os níveis municipal e estadual participem ativamente da vigilância dos casos para identificar os fatores determinantes no surgimento dos mesmos, e promover ações para melhorar a confiabilidade dos dados. Em suma, fazem-se necessários novos estudos que corroborem para o entendimento sobre o crescimento dos casos de HIV em pessoas idosas e que possam subsidiar ações específicas no campo. O fortalecimento da Política Nacional de Saúde da Pessoa Idosa faz parte do amplo espectro de desafios impostos ao poder público e, sobretudo, da luta da sociedade em defesa do SUS.

\section{REFERÊNCIAS}

1. AFFELDT AB, et al. Perfil de pessoas idosas vivendo com HIV/aids em Pelotas, sul do Brasil, 1998 a 2013. Epidemiol. Serv. Saúde, 2015; 24(1):79-86.

2. AGUIAR RB, et al. Conhecimento e atitudes sobre sexualidade em pessoas idosas com HIV. Ciência \& Saúde Coletiva [online]. V. 25, n. 6 [Acessado 16 Maio 2021], pp. 2051-2062. Disponível em: https://doi.org/10.1590/141381232020256.18432018. ISSN 1678-4561.

3. ALENCAR RA, et al. Repply: integrative review of literature: nursing care to aged people with HIV. Rev Bras Enferm. 2018; 71(Suppl 5): 2079-80.

4. BARBOZA, R. Homens idosos e o HIV/Aids no campo da Saúde Coletiva: vulnerabilidades e desafios na quarta década da epidemia. Periódicos Secretaria Estadual de Saúde de São Paulo, 2012.

5. BRASIL. Lei $n^{\circ}=10.741$, de $1^{\circ}$ de outubro de 2003. Dispõe sobre o Estatuto do Idoso e dá outras providências. Brasília: Presidência da República. 2003. Disponível em: http://www.planalto.gov.br/ccivil_03/leis/2003/l10.741.htm. Acesso em: 24/03/2021.

6. BRASIL. Infecção pelo HIV. Boletim HIV/AIDS. Secretaria Estadual de Saúde de Pernambuco, Secretaria Executiva de Vigilância em Saúde, Diretoria Geral de Controle de Doenças e Agravos, Gerência de Prevenção e Controle da Aids e outras DST. Ano 8, dez. 2018. Disponível em: http://www.aids.gov.br/pt-br/pub/2019/boletim-epidemiologicode-hivaids-2019. Acesso em: 24 de março de 2021.

7. BRASIL. Instituto Brasileiro de Geografia e Estatística (IBGE). Idosos indicam caminhos para uma melhor idade. 2019. Disponível em: https://censo2021.ibge.gov.br/2012-agencia-de-noticias/noticias/24036-idosos-indicamcaminhos-para-uma-melhor-idade.html. Acessado em: 24 de março de 2021.

8. CASSETÉ J, et al. HIV/aids em idosos: estigmas, trabalho e formação em saúde. Rev. Bras. Geriatr. Gerontol., Rio de Janeiro, 2016; 19(5): 733-744.

9. CELEDÔNIO LP, ANDRADE LC. Aids na terceira idade: Sentimentos, percepções e perspectivas de mulheres vivendo com HIV/AIDS. Serv. Soc. \& Saúde, Campinas, SP, 2014; 13(1): 47-60.

10. DORNELAS NETO J, et al. Doenças sexualmente transmissíveis em idosos: uma revisão sistemática. Ciênc. Saúde coletiva, 2015; 20(12): 3853-3864.

11. FERREIRA VHS, et al. Ageísmo, políticas públicas voltadas para população idosa e participação social. REAS/EJCH, Sup(42): e2816.

12. LAROQUE MF, et al. Sexualidade do idoso: comportamento para a prevenção de DST/AIDS. Ver. Gaúcha Enferm 2011; 32(4): 774-780.

13. MACHADO YY, et al. Representações dos profissionais de saúde sobre HIV/AIDS: comparação entre homens e mulhere. Saúde e pesqui. (Impr.), 2020. ID: biblio-1150491.

14. MACIEL M, LAGANÀ L. Older women's sexual desire problems: biopsychosocial factors impacting them and barriers to their clinical assessment. Biomed Res Int, 2014; 2014(2): 1-9.

15. MAIA DC, et al. Notification of cases of HIV/AIDS among the elderly in the state of Ceará: the historical sequence between 2005 and 2014. Rev Bras Geriatr Gerontol. 2018; 21(5): 542-52. 
16. MINICHIELLO V, et al. STI epidemiology in the global older population: emerging challenges. Perspect Public Health 2012; 132(4):178-181.

17. PEATE I. Human immunodeficiency virus and the older person.Br J Nurs 2007; 16(10): 606-610

18. PEREIRA B, et al. Fatores associados à infecção pelo HIV/AIDS entre adolescentes e adultos jovens matriculados em Centro de Testagem e Aconselhamento no Estado da Bahia, Brasil. Ciênc. saúde coletiva, 2014; 19(3): 747-758.

19. OLIVEIRA BLCA, et al. Associação da cor/raça aos indicadores de saúde para idosos no Brasil: um estudo baseado na Pesquisa Nacional por Amostra de Domicílios (2008). Cad. Saúde Pública, Rio de Janeiro, 2014; 30(7): 1-15.

20. SACCO RCCS, et al. Mortes por HIV/AIDS em adultos idosos no Distrito Federal: análise de 2007 a 2016 . DST - J bras Doenças Sex Transm, 2020;32. Disponível em: http://www.bjstd.org/html.php?id_artigo=288. Acesso em: 25 de março de 2021.

21. SILVA R, et al. A epidemia da aids no Brasil: Análise do Perfil atual. Rev enferm UFPE on line, Recife, 2013; 7(10): 6039-8.

22. SILVA AO, et al. HIV na terceira idade: repercussões nos domínios da vida e funcionamento familiar. Revista da Faculdade de Serviço Social da Universidade do Estado do Rio de Janeiro. Rio de Janeiro, 2017; 15(39): $129-154$.

23. SOARES KG, MENEGHEL SN. O silêncio da sexualidade em idosos dependentes. Ciência \& Saúde Coletiva [online]. V. 26, n. 01 [Acessado 14 Maio 2021], pp. 129-136. Disponível em: https://doi.org/10.1590/141381232020261.30772020. ISSN 1678-4561.

24. SZIRONY TA. Infection with HIV in the elderly population. J Gerontol Nurs 1999; 25(10): 25-31.

25. TAQUETTE SR, MEIRELLES ZV. Discriminação racial e vulnerabilidade às DST/Aids: um estudo com adolescentes negras. Physis Revista de Saúde Coletiva, Rio de Janeiro, 2013; 23(1): 129-142.

26. VALENTE G, et al. Atividades causadoras de HIV em idosos: Revisão Integrativa. Rev enferm UFPE on line. Recife, 2013; 7(8): 5323-5329.

27. VERAS RP, OLIVEIRA, M. Envelhecer no Brasil: a construção de um modelo de cuidado. Ciência \& Saúde Coletiva, 2018; 23(6):1929-1936.

28. VIANA P, et al. Aspectos Epidemiológicos, Clínicos e Evolutivos da Aids em idosos no Norte do Ceará. SANARE, Sobral, $2017 ; 16(2): 31-36$.

29. VIERA CPB, et al. Tendência de infecções por HIV/Aids: aspectos da ocorrência em idosos entre 2008 e 2018 . Esc Anna Nery, 2021; 25(2): e20200051. 